\title{
WEAK AMENABILITY OF CONVOLUTION BANACH ALGEBRAS ON COMPACT HYPERGROUPS
}

\author{
HojJatollah Samea
}

\begin{abstract}
In this paper we find necessary and sufficient conditions for weak amenability of the convolution Banach algebras $A(K)$ and $L^{2}(K)$ for a compact hypergroup $K$, together with their applications to convolution Banach algebras $L^{p}(K)(2 \leq p<\infty)$. It will further be shown that the convolution Banach algebra $A(G)$ for a compact group $G$ is weakly amenable if and only if $G$ has a closed abelian subgroup of finite index.
\end{abstract}

\section{Introduction}

Vrem ([9]) gave a definition of $A(K)$ for a compact hypergroup $K$ and prove that $A(K)$ is a Banach algebra with convolution product. This Banach algebra plays a key role throughout the paper.

The organization of this paper is as follows. The preliminaries and notations are given in Section 1. In Section 2 we state and prove a number of basic results on weak amenability of general Banach algebras which are needed for the rest of the paper. As an application we prove that for every compact abelian hypergroup $K$, the convolution Banach algebras $L^{p}(K)(1 \leq p<\infty)$, and $C(K)$ are weakly amenable. In Section 3 we show that the convolution Banach algebra $A(K)$ is weakly amenable if and only if $\sup _{\pi \in \widehat{K}} k_{\pi}\left(d_{\pi}-1\right)<\infty\left(d_{\pi}\right.$ is the dimension of representation $\pi$, and $k_{\pi}$ is the constant defined by $k_{\pi}=c_{\pi}^{-1}$, where $c_{\pi}=\int_{K}|\langle\pi(x) \xi, \xi\rangle|^{2} d \omega_{K}(x)$ for some $\xi$ in the representation space $H_{\pi}$ of $\pi$ with $\|\xi\|=1$, see the proof of Theorem 2.2 of [9]). Also we prove that the convolution Banach algebra $L^{2}(K)$ is weakly amenable if and only if the set $\{\pi \in \widehat{K}: \operatorname{dim} \pi \supsetneqq 1\}$ is finite. As a result it will further be shown that the convolution Banach algebra $A(G)$ for a compact group $G$ is weakly amenable if and only if $G$ is almost abelian (i.e., $G$ has a closed abelian subgroup of finite index). Furthermore, we prove that if $K$ is a compact hypergroup such that $x * y$ is a finite set for every $x, y \in K$, then the convolution Banach algebras $L^{p}(K)(2 \leq p<\infty)$ are weakly amenable if and only if $K$ is finite or abelian. An application of these results has enabled us to prove that if $K$ is an infinite

Received October 24, 2008; Revised July 24, 2009

2000 Mathematics Subject Classification. 43A20.

Key words and phrases. hypergroup, weak amenability, convolution Banach algebras. 
non-abelian compact hypergroup such that $x * y$ is a finite set for every $x, y \in K$, then the set $\{\pi \in \widehat{K}: \operatorname{dim} \pi \supsetneqq 1\}$ is infinite.

\section{Preliminaries}

For a Banach algebra $A$, an $A$-bimodule will always refer to a Banach $A$ bimodule $X$, that is a Banach space which is algebraically an $A$-bimodule, and for which there is a constant $C_{X} \geq 0$ such that $\|a . x\|,\|x . a\| \leq C_{X}\|a\|\|x\|$ $(a \in A, x \in X)$. A bounded linear map $D: A \rightarrow X$ is called an $X$-derivation, if for each $a, b \in A, D(a b)=D(a) \cdot b+a \cdot D(b)$. For every $x \in X$, we define $a d_{x}^{A}$ by $a d_{x}^{A}(a)=a . x-x . a(a \in A)$. It is easily seen that $a d_{x}^{A}$ is a derivation. Derivations of this form are called inner derivations. The set of all derivations from $A$ into $X$ is denoted by $\mathcal{Z}^{1}(A, X)$, and the set of all inner $X$-derivations is denoted by $\mathcal{B}^{1}(A, X)$. We denote by $\mathcal{H}^{1}(A, X)$ the difference space of $\mathcal{Z}^{1}(A, X)$ modulo $\mathcal{B}^{1}(A, X)$.

Let $A$ be a Banach algebra and $X$ be a Banach $A$-bimodule. Then the Banach space $X^{*}$ with the dual module multiplications given by

$$
(f a)(x)=f(a x),(a f)(x)=f(x a) \quad\left(a \in A, f \in X^{*}, x \in X\right),
$$

defines a Banach $A$-bimodule called the dual Banach $A$-bimodule $X^{*}$. A Banach algebra $A$ is called amenable if for each Banach $A$-bimodule $X, \mathcal{H}^{1}\left(A, X^{*}\right)$ $=0$. Every Banach algebra $A$ with the product of $A$ giving the two module multiplications defines a Banach $A$-bimodule. Let $A^{*}$ be the dual $A$-bimodule. A Banach algebra $A$ is called weakly amenable if $\mathcal{H}^{1}\left(A, A^{*}\right)=0$.

Let $H$ be a $n$-dimensional Hilbert space and $\mathcal{B}(H)$ be the space of all linear operators on $H$. Clearly we can identify $\mathcal{B}(H)$ with $\mathbb{M}_{n}(\mathbb{C})$ (the space of all $n \times$ $n$-matrices on $\mathbb{C})$. For $E \in \mathcal{B}(H)$, let $\left(\lambda_{1}, \ldots, \lambda_{n}\right)$ be the sequence of eigenvalues of the operator $|E|$, written in any order. Define $\|E\|_{\varphi_{\infty}}=\max _{1 \leq i \leq n}\left|\lambda_{i}\right|$, and $\|E\|_{\varphi_{p}}=\left(\sum_{i=1}^{n}\left|\lambda_{i}\right|^{p}\right)^{\frac{1}{p}}(1 \leq p<\infty)$. For more details see Definition D.37 and Theorem D.40 of [4].

Throughout this paper $K$ is a (measured) locally compact hypergroup with involution $x \mapsto \bar{x}$ and the identity $e$ as defined by Jewett $([5])$. By the term measured we mean that $K$ admits a left Haar measure $\omega_{K}$. Let $M(K)$ be the space of all bounded regular Borel measures on $K$. For $1 \leq p \leq \infty$, let $L^{p}(K)=L^{p}\left(K, \omega_{K}\right)$. For $x, y \in K$ define $x * y$ as the set $\operatorname{supp}\left(\varepsilon_{x} * \varepsilon_{y}\right)$. For Borel functions $f$ and $g$, at least one of which is $\sigma$-finite, define the convolution $f * g$ on $K$ by $(f * g)(x)=\int_{K} f(x * y) g(\bar{y}) d \omega_{K}(y)(x \in K)$, where $f(x * y)=$ $\int_{K} f d\left(\varepsilon_{x} * \varepsilon_{y}\right)$.

Let $K$ be a compact hypergroup. By Theorem 1.3.28 of [1], $K$ admits a left Haar measure. Throughout the present paper we use the normalized Haar measure $\omega_{K}$ on the compact hypergroup $K$ (i.e., $\omega_{K}(K)=1$ ). If $\pi \in \widehat{K}$ (where $\widehat{K}$ is the set of equivalence classes of continuous irreducible representations of $K$, c.f. [1], 11.3 of [5], and [9]), then by Theorem 2.2 of [9], $\pi$ is finite dimensional. Furthermore by the proof of Theorem 2.2 of [9], there exists a 
constant $c_{\pi}$ such that for each $\xi \in H_{\pi}$ with $\|\xi\|=1$

$$
\int_{K}|\langle\pi(x) \xi, \xi\rangle|^{2} d \omega_{K}(x)=c_{\pi} .
$$

Let $k_{\pi}=c_{\pi}^{-1}$. By Theorem 2.6 of [9], $k_{\pi} \geq d_{\pi}$. Moreover if $K$ is a group, then $k_{\pi}=d_{\pi}$. For each $\pi \in \widehat{K}$, let $H_{\pi}$ be the representation space of $\pi$ and $d_{\pi}=\operatorname{dim} H_{\pi}$. The *-algebra $\prod_{\pi \in \widehat{K}} \mathcal{B}\left(H_{\pi}\right)$ will denoted by $\mathfrak{E}(\widehat{K})$; scaler multiplication, addition, multiplication, and the adjoint of an element are defined coordinate-wise. Let $E=\left(E_{\pi}\right)$ be an element of $\mathfrak{E}(\widehat{K})$. We define $\|E\|_{p}:=\left(\sum_{\pi \in \widehat{K}} k_{\pi}\left\|E_{\pi}\right\|_{\varphi_{p}}^{p}\right)^{\frac{1}{p}}(1 \leq p<\infty)$, and $\|E\|_{\infty}=\sup _{\pi \in \widehat{K}}\left\|E_{\pi}\right\|_{\varphi_{\infty}}$. For $1 \leq p \leq \infty, \mathfrak{E}_{p}(\widehat{K})$ is defined as the set of all $E \in \mathfrak{E}(\widehat{K})$ for which $\|E\|_{p}<$ $\infty$. By Theorems $28.25,28.27$, and $28.32(\mathrm{v})$ of $[4]$, the spaces $\left(\mathfrak{E}_{p}(\widehat{K}),\|\cdot\|_{p}\right)$ $(1 \leq p \leq \infty)$ are Banach algebras. Let $\mu \in M(K)$. The set of all $E \in \mathfrak{E}(\widehat{K})$ such that $\left\{\pi \in \widehat{K}: E_{\pi} \neq 0\right\}$ is finite is denoted by $\mathfrak{E}_{00}(\widehat{K})$. The Fourier transform of $\mu$ at $\pi \in \widehat{K}$ is denoted by $\widehat{\mu}(\pi)$ and defined as the operator $\widehat{\mu}(\pi)=\int_{K} \overline{\pi(x)} d \mu(x)$ on $H_{\pi}$. Define $\widehat{\mu} \in \mathfrak{E}(\widehat{K})$ by $\widehat{\mu}_{\pi}=\widehat{\mu}(\pi) \in \mathcal{B}\left(H_{\pi}\right)$ (for more details see Theorem 3.2 of [9]). If $\pi \in \widehat{K}, \mathfrak{T}_{\pi}(K)$ is defined the set of all finite complex linear combinations of functions of the form $x \mapsto\langle\pi(x)(\xi), \eta\rangle$, where $\xi, \eta \in H_{\pi}$. Define $\mathfrak{T}(K)=\bigcup_{\pi \in \widehat{K}} \mathfrak{T}_{\pi}(K)$. Functions in $\mathfrak{T}(K)$ are called trigonometric polynomials on $K$. Clearly $\{\widehat{f}: f \in \mathfrak{T}(K)\}=\mathfrak{E}_{00}(\widehat{K})$ (see also Theorem 28.39 of [4] for the case of groups). If $f \in L^{1}(K)$, and $\sum_{\pi \in \widehat{K}} k_{\pi}\|\widehat{f}(\pi)\|_{\varphi_{1}}<\infty$, we say $f$ has an absolutely convergent Fourier series. The set of all functions with absolutely convergent Fourier series is denoted by $A(K)$ and called the Fourier space of $K$. For $f \in A(K)$ we define $\|f\|_{\varphi_{1}}=\|\widehat{f}\|_{1}$. By Proposition 4.2 of [9], $A(K)$ with the convolution product is a Banach algebra and isometrically isomorphic with $\mathfrak{E}_{1}(\widehat{K})$. Moreover each function $f \in A(K)$ can be regarded as the continuous function $\sum_{\pi \in \widehat{K}} k_{\pi} \operatorname{tr}(\widehat{f}(\pi) \pi(x))$. Also $\|f\|_{\infty} \leq\|f\|_{\varphi_{1}}$. However, $A(K)$ may not form a Banach algebra under point-wise product (see Example 4.12 of [9]).

\section{General results}

Our starting point of this section is the following proposition.

Proposition 2.1. Let $\mathfrak{A}$ be a Banach algebra. If there exists a family $\left(\mathfrak{A}_{\alpha}\right)_{\alpha}$ of amenable closed subalgebras of $\mathfrak{A}$ such that $\bigcup_{\alpha} \mathfrak{A}_{\alpha}$ is dense in $\mathfrak{A}$, then for each symmetric $\mathfrak{A}$-bimodule $X, \mathcal{Z}^{1}(\mathfrak{A}, X)=0$.

Proof. Let $D$ be a derivation from $\mathfrak{A}$ into $X$. If $a \in \mathfrak{A}$, then for each $\epsilon>0$, there exists $a_{\alpha} \in \mathfrak{A}_{\alpha}$ such that $\left\|a-a_{\alpha}\right\|<\frac{\epsilon}{\|D\|+1}$. Let $D_{\alpha}=D_{\mid \mathfrak{A}_{\alpha}}$. Clearly $D_{\alpha} \in \mathcal{Z}^{1}\left(\mathfrak{A}_{\alpha}, X\right)$. On one hand $\mathfrak{A}_{\alpha}$ is amenable, so by Proposition 1 of [3] there exists a net $\left(\xi_{\gamma}\right)_{\gamma}$ in $X$ such that $D_{\alpha}\left(a_{\alpha}^{\prime}\right)=\lim _{\gamma} a d_{\xi_{\gamma}}^{\mathfrak{A}_{\alpha}}\left(a_{\alpha}^{\prime}\right)$ for each $a_{\alpha}^{\prime} \in \mathfrak{A}_{\alpha}$. On the other hand since $X$ is a symmetric $\mathfrak{A}_{\alpha}$-bimodule, so for each $a_{\alpha}^{\prime} \in \mathfrak{A}_{\alpha}$ 
and for each $\gamma, a d_{\xi_{\gamma}}^{\mathfrak{A}_{\alpha}}\left(a^{\prime}\right)=0$. Therefore $D_{\alpha}=0$. Now, since $a_{\alpha} \in \mathfrak{A}_{\alpha}$, so

$$
\|D a\|=\left\|D a-D_{\alpha} a_{\alpha}\right\|=\left\|D\left(a-a_{\alpha}\right)\right\| \leq\|D\|\left\|a-a_{\alpha}\right\|<\epsilon .
$$

But $a \in \mathfrak{A}$ and $\epsilon>0$ are arbitrary, so $D=0$. It follows that $\mathcal{Z}^{1}(\mathfrak{A}, X)=0$.

Proposition 2.2. Let $\mathfrak{A}$ be a commutative Banach algebra. If there exists a family $\left(\mathfrak{A}_{\alpha}\right)_{\alpha}$ of weakly amenable closed subalgebras of $\mathfrak{A}$ such that $\bigcup_{\alpha} \mathfrak{A}_{\alpha}$ is dense in $\mathfrak{A}$, then $\mathfrak{A}$ is weakly amenable.

Proof. Let $D$ be a derivation from $\mathfrak{A}$ into $\mathfrak{A}^{*}$. For each $\alpha$ define $D_{\alpha}=D_{\mid \mathfrak{A}_{\alpha}}$. Since $\mathfrak{A}_{\alpha}$ is weakly amenable and $\mathfrak{A}^{*}$ is a symmetric $\mathfrak{A}_{\alpha}$-bimodule, so by Theorem 2.8.63(iii) of [2] $D_{\alpha}=0$. A method similar to the proof of Proposition 2.1 shows that $D=0$. Hence $\mathfrak{A}$ is weakly amenable.

Lemma 2.3. Let $K$ be a compact hypergroup and $\mathfrak{A}$ be any of Banach spaces $L^{p}(K)(1 \leq p<\infty), C(K)$, and $A(K)$. Then $\mathfrak{T}(K)$ is dense in $\mathfrak{A}$.

Proof. By Lemma 2.12 of [9], there is a net $\left(e_{\alpha}\right)$ in $\mathfrak{T}^{+}(K)$ such that $\left\|e_{\alpha}\right\|_{1}=1$, and $\left(e_{\alpha}\right)$ is a left approximate identity for $\left(L^{1}(K), *\right)$. Therefore $e_{\alpha} \longrightarrow \varepsilon_{e}$ in the weak* topology (or with the notations of [1], $\tau_{w}-\lim _{\alpha} e_{\alpha}=\varepsilon_{e}$ ). So by Lemma 1.4.6(ii) of [1], for each $1 \leq p<\infty$ and $f \in L^{p}(K),\left\|e_{\alpha} * f-f\right\|_{p} \longrightarrow 0$. Hence if $f \in L^{p}(K)$ and $\epsilon>0$, then there is $e_{\alpha} \in \mathfrak{T}(K)$ such that $\left\|e_{\alpha} * f-f\right\|_{p}<$ $\epsilon$. But $\widehat{e_{\alpha} * f}=\widehat{e_{\alpha}} \widehat{f} \in \mathfrak{E}_{00}(\widehat{K})=\widehat{\mathfrak{T}(K)}$. It follows that $\mathfrak{T}(K)$ is dense in $L^{p}(K)$. By Theorem 2.13 of $[9], \mathfrak{T}(K)$ is dense in $C(K)$. Since $\widehat{A(K)}=\mathfrak{E}_{1}(\widehat{K})$, and $\widehat{\mathfrak{T}(K)}=\mathfrak{E}_{00}(\widehat{K})$ is dense in $\mathfrak{E}_{1}(\widehat{K})$, so $\mathfrak{T}(K)$ is dense in $A(K)$.

Lemma 2.4. Let $K$ be a compact hypergroup and $\mathfrak{A}$ be any of convolution Banach algebras $L^{p}(K)(1 \leq p<\infty), A(K)$, and $C(K)$. Then there exists a family $\left(\mathfrak{A}_{\alpha}\right)_{\alpha}$ of amenable closed subalgebras of $\mathfrak{A}$ such that $\bigcup_{\alpha} \mathfrak{A}_{\alpha}$ is dense in $\mathfrak{A}$.

Proof. Let $\mathcal{F}=\{F \subseteq \widehat{K}: F$ is finite $\}$. For each $F \in \mathcal{F}$, define $\mathfrak{T}_{F}(K)$, through

$$
\widehat{\mathfrak{T}_{F}(K)}=\left\{E \in \mathfrak{E}(\widehat{K}): E_{\pi}=0 \text { for } \pi \notin F\right\} .
$$

Clearly $\widehat{\mathfrak{T}_{F}(K)}$ is a finite dimensional subalgebra of $\mathfrak{E}(\widehat{K})$, and is Banach algebraically isomorphic with $\ell^{\infty}-\oplus_{\pi \in F} \mathbb{M}_{d_{\pi}}(\mathbb{C})$. Hence by Exercise 4.1.3 of [7], $\widehat{\mathfrak{T}_{F}(K)}$ is amenable. Since $\mathfrak{T}_{F}(K) \subseteq \mathfrak{T}(K) \subseteq \mathfrak{A}$, so $\mathfrak{T}_{F}(K)$ is an amenable closed subalgebra of $\mathfrak{A}$. Clearly $\mathfrak{T}(K)=\bigcup_{F \in \mathcal{F}} \mathfrak{T}_{F}(K)$. Now, by Lemma 2.3, $\mathfrak{T}(K)$ is dense in $\mathfrak{A}$. This finishes the proof.

Proposition 2.5. Let $K$ be a compact hypergroup and $\mathfrak{A}$ be any of the convolution Banach algebras $L^{p}(K)(1 \leq p<\infty), A(K)$, and $C(K)$. Then for each symmetric Banach $\mathfrak{A}$-bimodule $X, \mathcal{H}^{1}(\mathfrak{A}, X)=0$.

Proof. This follows immediately from Lemma 2.4 and Proposition 2.1.

A combination of Lemma 2.4 and Proposition 2.2 yields the following result. 
Theorem 2.6. Let $K$ be a compact commutative hypergroup and $\mathfrak{A}$ be any of the convolution Banach algebras $L^{p}(K) 1 \leq p<\infty, A(K)$, and $C(K)$. Then $\mathfrak{A}$ is weakly amenable.

Remark 2.7. Example 4.10 of [8], shows that there exists a noncompact commutative hypergroup $K$ such that $L^{1}(K)$ is not weakly amenable. But, by Proposition 2.6 for a compact commutative hypergroup $K, L^{1}(K)$ is weakly amenable.

\section{Weak amenability of certain convolution Banach algebras on compact hypergroups}

The following proposition which is interesting in its own right, is needed in the sequel.

Proposition 3.1. Let $n \in \mathbb{N}$ and $1 \leq p, q \leq \infty$. If $D$ is a derivation from $\left(\mathbb{M}_{n}(\mathbb{C}),\|\cdot\|_{\varphi_{p}}\right)$ into $\left(\mathbb{M}_{n}(\mathbb{C}),\|\cdot\|_{\varphi_{q}}\right)$, then there exists $E \in \mathbb{M}_{n}(\mathbb{C})$ such that

$$
D(A)=A E-E A \quad\left(A \in \mathbb{M}_{n}(\mathbb{C})\right),
$$

and $\|E\|_{\varphi_{q}} \leq n\|D\|$. If $n=1$, we can take $E=0$.

Proof. For each $1 \leq i, j \leq n$, let $\mathcal{E}_{i j}$ be the elementary $n \times n$-matrix defined as follows: $\left(\mathcal{E}_{i j}\right)_{k l}=1$ for $k=i, l=j$ and $\left(\mathcal{E}_{i j}\right)_{k l}=1$ for all others $1 \leq k, l \leq n$. Let

$$
E=\frac{1}{n} \sum_{i, j=1}^{n} \mathcal{E}_{i j} D\left(\mathcal{E}_{j i}\right)
$$

By an elementary calculation one can prove that for each $A \in \mathbb{M}_{n}(\mathbb{C}), D(A)=$ $A E-E A$ (see also Proposition 1.9.20 and the proof of Theorem 1.9.21((b) $\Rightarrow(\mathrm{a})$ ) of [2]). By Theorem D.52 of [4]

$$
\begin{aligned}
\|E\|_{\varphi_{q}} & \leq \frac{1}{n} \sum_{i, j=1}^{n}\left\|\mathcal{E}_{i j} D\left(\mathcal{E}_{j i}\right)\right\|_{\varphi_{q}} \leq \frac{1}{n} \sum_{i, j=1}^{n}\left\|\mathcal{E}_{i j}\right\|_{\varphi_{q}}\left\|D\left(\mathcal{E}_{j i}\right)\right\|_{\varphi_{q}} \\
& \leq \frac{1}{n} \sum_{i, j=1}^{n}\left\|\mathcal{E}_{i j}\right\|_{\varphi_{q}}\|D\|\left\|\mathcal{E}_{j i}\right\|_{\varphi_{p}}=\frac{1}{n} \sum_{i, j=1}^{n}\|D\|=n\|D\| .
\end{aligned}
$$

If $n=1$, then clearly $D=0$, and so we can take $E=0$.

Lemma 3.2. Let $K$ be a compact hypergroup. Then the mapping $\Theta: \mathfrak{E}_{\infty}(\widehat{K}) \rightarrow$ $A(K)^{*}$ given by

$$
\langle f, \Theta(A)\rangle=\sum_{\pi \in \widehat{G}} k_{\pi} \operatorname{tr}\left(\widehat{f}(\pi) A_{\pi}\right) \quad\left(f \in A(K), A \in \mathfrak{E}_{\infty}(\widehat{K})\right),
$$

is an isometrically Banach $A(K)$-bimodule isomorphism. In particular

$$
\Theta(\widehat{f} A)=f \cdot \Theta(A), \Theta(A \widehat{f})=\Theta(A) \cdot f \quad\left(f \in A(K), A \in \mathfrak{E}_{\infty}(\widehat{K})\right) .
$$


Proof. By Proposition 4.2 of [9], the mapping $\mathcal{F}: A(K) \rightarrow \mathfrak{E}_{1}(\widehat{K}), f \mapsto \widehat{f}$ is an isometrically algebra isomorphism. So the adjoint $\mathcal{F}^{*}: \mathfrak{E}_{1}(\widehat{K})^{*} \rightarrow A(K)^{*}$ of $\mathcal{F}$ is an isometrically Banach space isomorphism. It is obvious that

$$
\mathcal{F}^{*}(\widehat{f} . E)=f . \mathcal{F}^{*}(E), \mathcal{F}^{*}(E . \widehat{f})=\mathcal{F}^{*}(E) . f \quad\left(f \in A(K), E \in \mathfrak{E}_{1}(\widehat{K})^{*}\right) .
$$

By Theorem 28.31 of [4], the mapping $T: \mathfrak{E}_{\infty}(\widehat{K}) \rightarrow \mathfrak{E}_{1}(\widehat{K})^{*}$ given by

$$
\langle B, T(A)\rangle=\sum_{\pi \in \widehat{K}} k_{\pi} \operatorname{tr}\left(B_{\pi} A_{\pi}\right) \quad\left(A \in \mathfrak{E}_{1}(\widehat{K}), B \in \mathfrak{E}_{\infty}(\widehat{K})\right),
$$

is an isometrically Banach space isomorphism. For each $A, B \in \mathfrak{E}_{1}(\widehat{K})$, and $X \in \mathfrak{E}_{\infty}(\widehat{K})$, we have

$$
\begin{aligned}
\langle B, T(X) \cdot A\rangle & =\langle A B, T(X)\rangle=\sum_{\pi \in \widehat{G}} k_{\pi} \operatorname{tr}\left((A B)_{\pi} X_{\pi}\right) \\
& \left.=\sum_{\pi \in \widehat{G}} k_{\pi} \operatorname{tr}\left(X_{\pi}(A B)_{\pi}\right)\right)=\sum_{\pi \in \widehat{G}} k_{\pi} \operatorname{tr}\left((X A)_{\pi} B_{\pi}\right)=\langle B, T(X A)\rangle .
\end{aligned}
$$

So $T(X) \cdot A=T(X A)$. Similarly $A \cdot T(X)=T(A X)$. Now, since $\Theta=\mathcal{F}^{*} \circ T$, so it is an isometrically Banach space isomorphism, and moreover

$$
\Theta(\widehat{f} A)=f . \Theta(A), \Theta(A \widehat{f})=\Theta(A) \widehat{f} \quad\left(f \in A(K), A \in \mathfrak{E}_{\infty}(\widehat{K})\right) .
$$

Theorem 3.3. Let $K$ be a compact hypergroup. Then the convolution Banach algebra $A(K)$ is weakly amenable if and only if $\sup _{\pi \in \widehat{K}} k_{\pi}\left(d_{\pi}-1\right)<\infty$.

Proof. By Lemma 3.2, the convolution Banach algebra $A(K)$ is weakly amenable if and only if each derivation from $\mathfrak{E}_{1}(\widehat{K})$ into $\mathfrak{E}_{\infty}(\widehat{K})$ is inner.

Suppose $\sup _{\pi \in \widehat{K}} k_{\pi}\left(d_{\pi}-1\right)<\infty$. Let $D \in \mathcal{Z}^{1}\left(\mathfrak{E}_{1}(\widehat{K})\right.$, $\left.\mathfrak{E}_{\infty}(\widehat{K})\right)$. For each $\pi \in \widehat{K}$, and $A \in B\left(H_{\pi}\right)$, define $A^{\pi} \in \mathfrak{E}(I)$ as follows: $\left(A^{\pi}\right)_{\pi}=A$ and $\left(A^{\pi}\right)_{\sigma}=0$ for $\sigma \neq \pi$. Define $D_{\pi}: B\left(H_{\pi}\right) \rightarrow B\left(H_{\pi}\right)$ through

$$
D_{\pi}(A)=\left(D\left(A^{\pi}\right)\right)_{\pi} \quad\left(A \in B\left(H_{\pi}\right)\right) .
$$

Clearly $D_{\pi}$ is a derivation from $\left(B\left(H_{\pi}\right),\|\cdot\|_{\varphi_{1}}\right)$ into $\left(B\left(H_{\pi}\right),\|\cdot\|_{\varphi_{\infty}}\right)$. So by Proposition 3.1, there exists $E(\pi) \in B\left(H_{\pi}\right)$ such that $\|E(\pi)\|_{\varphi_{\infty}} \leq d_{\pi}\left\|D_{\pi}\right\|$, and

$$
D\left(A_{\pi}\right)=A_{\pi} E(\pi)-E(\pi) A_{\pi} \quad\left(A_{\pi} \in B\left(H_{\pi}\right)\right) .
$$

Further if $d_{\pi}=1$, we can assume $E(\pi)=0$. By definition of $\|\cdot\|_{\varphi_{1}}$, if $d_{\pi} \neq 1$, then

$$
\begin{aligned}
\|E(\pi)\|_{\varphi_{\infty}} & \leq d_{\pi}\left\|D_{\pi}\right\|=d_{\pi} \sup _{\|A\|_{\varphi_{1}}=1}\left\|D_{\pi}(A)\right\|_{\varphi_{\infty}} \\
& =d_{\pi} \sup _{\|A\|_{\varphi_{1}}=1}\left\|\left(D\left(A^{\pi}\right)\right)_{\pi}\right\|_{\varphi_{\infty}} \leq d_{\pi} \sup _{\|A\|_{\varphi_{1}}=1}\left\|D\left(A^{\pi}\right)\right\|_{\infty} \\
& \leq d_{\pi}\|D\| \sup _{\|A\|_{\varphi_{1}}=1}\left\|A^{\pi}\right\|_{1}=d_{\pi} k_{\pi}\|D\| \leq k_{\pi}^{2}\|D\| .
\end{aligned}
$$


Now, if $E \in \mathfrak{E}(\widehat{K})$ is defined by $E_{\pi}=E(\pi)(\pi \in \widehat{K})$, then

$$
\|E\|_{\infty}=\sup _{\pi \in \widehat{K}}\left\|E_{\pi}\right\|_{\varphi_{\infty}} \leq\|D\| \sup _{\pi \in \widehat{K}, d_{\pi} \neq 1} k_{\pi}^{2} \leq\|D\| \sup _{\pi \in \widehat{K}}\left(k_{\pi}\left(d_{\pi}-1\right)\right)^{2}<\infty,
$$

and so $E \in \mathfrak{E}_{\infty}(\widehat{K})$. So if $I_{\pi}$ is the $d_{\pi} \times d_{\pi^{-}}$-identity matrix, then by Proposition 1.8.2(ii) of [2], $D\left(I_{\pi}^{\pi}\right)=0$. Hence

$$
\begin{aligned}
D(A)_{\pi} & =\left(D(A) I_{\pi}^{\pi}\right)_{\pi}=\left(D\left(A I_{\pi}^{\pi}\right)-A D\left(I_{\pi}^{\pi}\right)\right)_{\pi}=\left(D\left(A I_{\pi}^{\pi}\right)\right)_{\pi} \\
& =\left(D\left(A_{\pi}^{\pi}\right)\right)_{\pi}=D_{\pi}\left(A_{\pi}\right)=A_{\pi} E(\pi)-E(\pi) A_{\pi}=(A E-E A)_{\pi} .
\end{aligned}
$$

So $D(A)=A E-E A$. It follows that the convolution Banach algebra $A(K)$ is weakly amenable.

Now, suppose $\sup _{\pi \in \widehat{K}} k_{\pi}\left(d_{\pi}-1\right)=\infty$. Define $E \in \mathfrak{E}(\widehat{K})$ by $E_{\pi}=k_{\pi} \mathcal{E}_{11}^{\pi}$, where $\mathcal{E}_{11}^{\pi}$ is the elementary $d_{\pi} \times d_{\pi}$-matrix that defined in the proof of Proposition 3.1. Then $\left\|E_{\pi}\right\|_{\varphi_{\infty}}=k_{\pi}(\pi \in \widehat{K})$, and $E \notin \mathfrak{E}_{\infty}(\widehat{K})$. Define $D: \mathfrak{E}_{1}(\widehat{K}) \rightarrow$ $\mathfrak{E}_{\infty}(\widehat{K})$ by $D(A)=A E-E A\left(A \in \mathfrak{E}_{1}(\widehat{K})\right)$. For $A \in \mathfrak{E}_{1}(\widehat{K})$, use Theorems D.51(i) and D.52(i) of [4] to write

$$
\begin{aligned}
\|D(A)\|_{\infty} & =\|A E-E A\|_{\infty}=\sup _{\pi \in \widehat{K}}\left\|A_{\pi} E_{\pi}-E_{\pi} A_{\pi}\right\|_{\varphi_{\infty}} \\
& \leq \sup _{\pi \in \widehat{K}}\left(\left\|A_{\pi} E_{\pi}\right\|_{\varphi_{\infty}}+\left\|E_{\pi} A_{\pi}\right\|_{\varphi_{\infty}}\right) \leq 2 \sup _{\pi \in \widehat{K}}\left\|E_{\pi}\right\|_{\varphi_{\infty}}\left\|A_{\pi}\right\|_{\varphi_{\infty}} \\
& =2 \sup _{\pi \in \widehat{K}} k_{\pi}\left\|A_{\pi}\right\|_{\varphi_{\infty}} \leq 2 \sup _{\pi \in \widehat{K}} k_{\pi}\left\|A_{\pi}\right\|_{\varphi_{1}} \leq 2 \sum_{\pi \in \widehat{K}} k_{\pi}\left\|A_{\pi}\right\|_{\varphi_{1}} \\
& =2\|A\|_{1} .
\end{aligned}
$$

Therefore $D$ is well-defined and continuous. Clearly $D$ is a derivation. We claim that $D$ is not inner. Suppose to the contrary that $D$ is inner. Then there exists $E^{\prime} \in \mathfrak{E}_{\infty}(\widehat{K})$ such that for each $A \in \mathfrak{E}_{1}(\widehat{K}), D(A)=A E^{\prime}-E^{\prime} A$ or equivalently $A\left(E-E^{\prime}\right)=\left(E-E^{\prime}\right) A$. So for each $\pi \in \widehat{K}$ and $A_{\pi} \in B\left(H_{\pi}\right)$, $A_{\pi}\left(E_{\pi}-E_{\pi}^{\prime}\right)=\left(E_{\pi}-E_{\pi}^{\prime}\right) A_{\pi}$. Hence by Corollary 27.10 of [4], there exists $\lambda_{\pi} \in \mathbb{C}$ such that $E_{\pi}-E_{\pi}^{\prime}=\lambda_{\pi} I_{\pi}$. Since $\sup _{\pi \in \widehat{K}} k_{\pi}\left(d_{\pi}-1\right)=\infty$, so there exists a subset $\left\{\pi_{n}: n \in \mathbb{N}\right\}$ of $\widehat{K}$ such that $\pi_{m} \neq \pi_{n}$ for $m \neq n, d_{\pi_{n}} \gtreqless 1$ for $n \in \mathbb{N}$, and $\lim _{n} k_{\pi_{n}}\left(d_{\pi_{n}}-1\right)=\infty$. The eigenvalues of $\left|E_{\pi_{n}}-\lambda_{\pi_{n}} I_{\pi_{n}}\right|$ are $\left|\lambda_{\pi_{n}}\right|$ with multiplicity $d_{\pi_{n}}-1$, and $\left|k_{\pi_{n}}-\lambda_{\pi_{n}}\right|$ with multiplicity 1 . So

$$
\left\|E_{\pi_{n}}^{\prime}\right\|_{\varphi_{\infty}}=\max \left\{\left|\lambda_{\pi_{n}}\right|,\left|k_{\pi_{n}}-\lambda_{\pi_{n}}\right|\right\} \geq \frac{1}{2} k_{\pi_{n}}
$$

and hence

$$
\begin{aligned}
\left\|E^{\prime}\right\|_{\infty} & \geq \sup _{n \in \mathbb{N}}\left\|E_{\pi_{n}}^{\prime}\right\|_{\varphi_{\infty}} \geq \frac{1}{2} \sup _{n \in \mathbb{N}} k_{\pi_{n}} \geq \frac{1}{2} \sup _{n \in \mathbb{N}}\left(k_{\pi_{n}}\left(d_{\pi_{n}}-1\right)\right)^{\frac{1}{2}} \\
& =\frac{1}{2} \lim _{n}\left(k_{\pi_{n}}\left(d_{\pi_{n}}-1\right)\right)^{\frac{1}{2}}=\infty .
\end{aligned}
$$

That is $E^{\prime} \notin \mathfrak{E}_{\infty}(\widehat{K})$. This contradiction proves our claim. Hence the convolution Banach algebra $A(K)$ is not weakly amenable. 
Theorem 3.4. Let $G$ be a compact group. Then the convolution Banach algebra $A(G)$ is weakly amenable if and only if $G$ has a closed abelian subgroup of finite index.

Proof. Since $G$ is a compact group, so $k_{\pi}=d_{\pi}(\pi \in \widehat{G})$. Hence, by Theorem 3.3 , the convolution Banach algebra $A(G)$ is weakly amenable if and only if $\sup _{\pi \in \widehat{G}} d_{\pi}<\infty$. By [6], it is valid if and only if $G$ has a closed abelian subgroup of finite index.

Remark 3.5. A locally compact group $G$ is called almost abelian, if it has a closed abelian subgroup of finite index. Hence for a compact group $G$, the convolution Banach algebra $A(G)$ is weakly amenable if and only if $G$ is almost abelian.

Proposition 3.6. Let $K$ be a compact hypergroup. Then the convolution Banach algebra $L^{2}(K)$ is weakly amenable if and only if the set $\{\pi \in \widehat{K}: \operatorname{dim} \pi \supsetneqq$ 1\} is finite.

Proof. Note that by Theorem 3.4 of [9], the convolution Banach algebra $L^{2}(K)$ is isometrically algebra isomorphic with $\mathfrak{E}_{2}(\widehat{K})$. Now, by Theorem 34.35 of [4], and an argument as Lemma 3.2, the mapping $T: \mathfrak{E}_{2}(\widehat{K}) \rightarrow \mathfrak{E}_{2}(\widehat{K})^{*}$ given by

$$
\langle B, T(A)\rangle=\sum_{\pi \in \widehat{K}} k_{\pi} \operatorname{tr}\left(B_{\pi} A_{\pi}\right) \quad\left(A \in \mathfrak{E}_{2}(\widehat{K}), B \in \mathfrak{E}_{2}(\widehat{K})\right),
$$

defines a Banach $\mathfrak{E}_{2}(\widehat{K})$-bimodule isomorphism between $\mathfrak{E}_{2}(\widehat{K})^{*}$ and $\mathfrak{E}_{2}(\widehat{K})$, where the product of $\mathfrak{E}(\widehat{K})$ giving the two module multiplications. It follows that the convolution Banach algebra $L^{2}(K)$ is weakly amenable if and only if each derivation from $\mathfrak{E}_{2}(\widehat{K})$ into $\mathfrak{E}_{2}(\widehat{K})$ is inner.

Suppose that the set $\{\pi \in \widehat{K}: \operatorname{dim} \pi \supsetneqq 1\}$ is finite. Let $D \in \mathcal{Z}^{1}\left(\mathfrak{E}_{2}(\widehat{K})\right.$, $\left.\mathfrak{E}_{2}(\widehat{K})\right)$. With the notations of the proof of Theorem 3.3, define $D_{\pi}: B\left(H_{\pi}\right) \rightarrow$ $B\left(H_{\pi}\right)$ through

$$
D_{\pi}(A)=\left(D\left(A^{\pi}\right)\right)_{\pi} \quad\left(A \in B\left(H_{\pi}\right)\right) .
$$

Clearly $D_{\pi}$ is a derivation from $\left(B\left(H_{\pi}\right),\|\cdot\|_{\varphi_{2}}\right)$ into $\left(B\left(H_{\pi}\right),\|\cdot\|_{\varphi_{2}}\right)$. So by Proposition 3.1, there exists $E(\pi) \in B\left(H_{\pi}\right)$ such that $\|E(\pi)\|_{\varphi_{2}} \leq d_{\pi}\left\|D_{\pi}\right\|$, and

$$
D\left(A_{\pi}\right)=A_{\pi} E(\pi)-E(\pi) A_{\pi} \quad\left(A_{\pi} \in B\left(H_{\pi}\right)\right) .
$$

Further if $d_{\pi}=1$, we can assume $E(\pi)=0$. Since the set $\{\pi \in \widehat{K}: \operatorname{dim} \pi \supsetneqq 1\}$ is finite, so $E \in \mathfrak{E}_{2}(\widehat{K})$. An argument as in the proof of Theorem 3.3, shows that $D(A)=A E-E A\left(A \in \mathfrak{E}_{2}(\widehat{K})\right)$, and so $D$ is inner. It follows that the convolution Banach algebra $L^{2}(K)$ is weakly amenable.

Now, suppose that the set $\{\pi \in \widehat{K}: \operatorname{dim} \pi \supsetneqq 1\}$ is infinite. Define $E \in \mathfrak{E}(\widehat{K})$ by $E_{\pi}=\mathcal{E}_{11}^{\pi}(\pi \in \widehat{K})$, and $D: \mathfrak{E}_{2}(\widehat{K}) \rightarrow \mathfrak{E}_{2}(\widehat{K})$ by $D(A)=A E-E A$ $\left(A \in \mathfrak{E}_{2}(\widehat{K})\right)$. Then by a method similar to the proof of Theorem 3.3, one can prove that $D$ is a derivation. Since the set $\{\pi \in \widehat{K}: \operatorname{dim} \pi \supsetneqq 1\}$ is infinite, so 
there exists a subset $\left\{\pi_{n}: n \in \mathbb{N}\right\}$ of $\widehat{K}$ such that $\pi_{m} \neq \pi_{n}$ for $m \neq n$, and $d_{\pi_{n}} \gtreqless 1$ for $n \in \mathbb{N}$. Let $E^{\prime} \in \mathfrak{E}(\widehat{K})$ such that $D(A)=A E^{\prime}-E^{\prime} A\left(A \in \mathfrak{E}_{2}(\widehat{K})\right)$. By an argument similar to the proof of Theorem 3.3, it can be proved that for each $n \in \mathbb{N},\left\|E_{\pi_{n}}^{\prime}\right\|_{\varphi_{2}} \geq \frac{1}{2}$. Hence

$$
\left\|E^{\prime}\right\|_{2}^{2}=\sum_{\pi \in \widehat{K}} k_{\pi}\left\|E^{\prime}\right\|_{\varphi_{2}}^{2} \geq \sum_{n=1}^{\infty} k_{\pi}\left(\frac{1}{2}\right)^{2}=\infty,
$$

and so $E^{\prime} \notin \mathfrak{E}_{2}(\widehat{K})$. It follows that $D$ is not inner, and so the convolution Banach algebra $L^{2}(K)$ is not weakly amenable.

By a method similar to the above proposition we have the following result.

Proposition 3.7. Let $K$ be a compact hypergroup. Then each derivation from the convolution Banach algebra $A(K)$ into itself is inner if and only if the set $\{\pi \in \widehat{K}: \operatorname{dim} \pi \supsetneqq 1\}$ is finite.

Proposition 3.8. Let $K$ be a compact hypergroup such that $x * y$ is a finite set for each $x, y \in K$. If $2 \leq p<\infty$, then the convolution Banach algebra $L^{p}(K)$ is weakly amenable if and only if $K$ is finite or abelian.

Proof. Suppose $K$ is infinite and non-abelian. There exist $x, y \in K$ such that $\varepsilon_{x} * \varepsilon_{y} \neq \varepsilon_{y} * \varepsilon_{x}$. Since $2 \leq p<\infty$, so if $\frac{1}{p}+\frac{1}{q}=1$, then $p \geq q$. Hence $L^{p}(K) \subseteq L^{q}(K)$, and so the mapping

$$
D_{x}: L^{p}(K) \rightarrow L^{q}(K), f \mapsto \varepsilon_{x} * f-f * \varepsilon_{x},
$$

gives a well-defined derivation. We claim that $D_{x}$ is non-inner. Suppose to the contrary that $D_{x}=a d_{g}$ for some $g \in L^{q}(K)$. Then for each $f \in L^{p}(K)$, $f *\left(\varepsilon_{x}-g\right)=\left(\varepsilon_{x}-g\right) * f$. Since $L^{p}(K)$ is dense in $L^{1}(K)$, so for each $f \in L^{1}(K)$, $f *\left(\varepsilon_{x}-g\right)=\left(\varepsilon_{x}-g\right) * f$. Let $\left(e_{\alpha}\right)$ be a bounded approximate identity for $L^{1}(K)$. Then with respect to the weak*-topology $\tau_{w}$ on $M(K)$

$$
\begin{aligned}
\varepsilon_{x} * \varepsilon_{y}-\varepsilon_{y} * \varepsilon_{x} & =\tau_{w}-\lim _{\alpha}\left(\varepsilon_{x} *\left(e_{\alpha} * \varepsilon_{y}\right)-\left(e_{\alpha} * \varepsilon_{y}\right) * \varepsilon_{x}\right) \\
& =\tau_{w}-\lim _{\alpha} D_{x}\left(e_{\alpha} * \varepsilon_{y}\right)=\tau_{w}-\lim _{\alpha} a d_{g}\left(e_{\alpha} * \varepsilon_{y}\right) \\
& =g * \varepsilon_{y}-\varepsilon_{y} * g \in L^{q}(K) \subseteq L^{1}(K) .
\end{aligned}
$$

Using the fact that $\varepsilon_{x} * \varepsilon_{y} \neq \varepsilon_{y} * \varepsilon_{x}$, and $x * y, y * x$ are finite, we conclude that $\varepsilon_{x} * \varepsilon_{y}-\varepsilon_{y} * \varepsilon_{x}$ is a non-zero discrete measure. Since $K$ is compact and infinite, it is not discrete. Therefore $\varepsilon_{x} * \varepsilon_{y}-\varepsilon_{y} * \varepsilon_{x} \notin L^{1}(K)$. This contradiction shows that $D_{x}$ is non-inner. Now, it is easy to check that the well-known isometrical Banach space isomorphism $T: L^{q}(K) \rightarrow L^{p}(K)^{*}$, that given by

$$
\langle g, T(f)\rangle=\int_{K} f(x) g(x) d x \quad\left(f \in L^{q}(K), g \in L^{p}(K)\right),
$$

defines an $L^{p}(K)$-module homomorphism. Hence the convolution Banach algebra $L^{p}(K)$ is not weakly amenable. 
If $K$ is abelian, then by Proposition 2.6, the convolution Banach algebra $L^{p}(K)$ is weakly amenable. If $K$ is a finite hypergroup, then $L^{p}(K)=\ell^{1}(K)=$ $C(K)=A(K)$, and $\widehat{K}$ is finite. Hence

$$
L^{p}(K) \cong \widehat{A(K)}=\mathfrak{E}_{1}(\widehat{K}) \cong \ell^{\infty}-\oplus_{\pi \in \widehat{K}} \mathbb{M}_{d_{\pi}}(\mathbb{C}),
$$

and so by Exercise 4.1.3 of [7], the convolution Banach algebra $L^{p}(K)$ is amenable, and in particular is weakly amenable.

Corollary 3.9. Let $K$ be a compact hypergroup such that $x * y$ is a finite set for each $x, y \in K$. Then the set $\{\pi \in \widehat{K}: \operatorname{dim} \pi \supsetneqq 1\}$ is finite if and only if $K$ is finite or abelian.

Proof. Clearly if $K$ is finite or abelian, then $\{\pi \in \widehat{K}: \operatorname{dim} \pi \supsetneqq 1\}$ is finite.

Let $K$ be infinite and non-abelian. Then by Proposition 3.8 the convolution Banach algebra $L^{2}(K)$ is not weakly amenable. Hence by Theorem 3.6, the set $\{\pi \in \widehat{K}: \operatorname{dim} \pi \supsetneqq 1\}$ is infinite.

We close this paper with the following open problems.

\section{Some open problems:}

1 ) Is $L^{1}(K)$ is weakly amenable for each compact hypergroup?

2 ) Is $L^{1}(K)$ is amenable for each compact commutative hypergroup? Is this true for each compact hypergroup?

3) What about the case $1 \leq p<2$ for Proposition 3.8?

4) Is Corollary 3.9 is valid for each compact hypergroup?

Acknowledgement. The author would like to thank the referee for invaluable comments. The author also would like to thank the University of Bu-Ali Sina (Hamedan) for its support.

\section{References}

[1] W. R. Bloom and H. Heyer, Harmonic Analysis of Probability Measures on Hypergroups, de Gruyter Studies in Mathematics, 20. Walter de Gruyter \& Co., Berlin, 1995.

[2] H. G. Dales, Banach Algebras and Automatic Continuity, London Mathematical Society Monographs. New Series, 24. Oxford Science Publications. The Clarendon Press, Oxford University Press, New York, 2000.

[3] F. Gordeau, Amenability of Lipschitz algebras, Math. Proc. Cambridge Philos. Soc. 112 (1992), no. 3, 581-588.

[4] E. Hewitt and K. A. Ross, Abstract harmonic analysis. Vol. II: Structure and analysis for compact groups. Analysis on locally compact Abelian groups, Springer-Verlag, New York-Berlin, 1970.

[5] R. I. Jewett, Spaces with an abstract convolution of measures, Advances in Math. 18 (1975), no. 1, 1-101.

[6] C. C. Moore, Groups with finite dimensional irreducible representations, Trans. Amer. Math. Soc. 166 (1972), 401-410.

[7] V. Runde, Lectures on Amenability, Lecture Notes in Mathematics, 1774. Springer-Verlag, Berlin, 2002.

[8] M. Skantharajah, Amenable hypergroups, Illinois J. Math. 36 (1992), no. 1, 15-46. 
[9] R. C. Vrem, Harmonic analysis on compact hypergroups, Pacific J. Math. 85 (1979), no. $1,239-251$

Department of Mathematics

Bu-Ali Sina University

HAMEDAN, IRAN

E-mail address: h.samea@basu.ac.ir 